\title{
Research on the Behavior Simulation Of Vehicles in Driving Simulator Under the Influence of Terrain
}

\author{
LiaoJinmin $^{1, a}$, WanGang ${ }^{2, b}$, LiFeng $^{3, c}$ \\ ${ }^{1}$ Information Engineering University; Zhengzhou Henan,450052, China \\ ${ }^{2}$ Information Engineering University; Zhengzhou Henan,450052, China \\ ${ }^{3}$ Information Engineering University; Zhengzhou Henan,450052, China \\ aemail: liaojinmin0413@126.com, bemail:806617147@qq.com, cemail:touch0413@126.com
}

Keywords: driving simulator ; terrain influence; physic engine; behavior simulation;

\begin{abstract}
Driving simulator needs to not only build a realistic simulated environment but also simulated the vehicle mobile behavior, which make its movement be in line with the real physical laws. The motion of the vehicles is not isolated, but influenced by the terrain. Therefore, to simulate the vehicle motion vividly, analyzing the influence of terrain on the vehicle motion is needed. Using the physics engine to build the realistic vehicle model which has its own physical property can make the vehicle move in line with the real physical laws. Combining the physics engine and graph rendering engine to build a virtual environment which contain physical space and the corresponding graphics space can display the complicated motion of vehicle under different terrain in line with the physical laws in real world.
\end{abstract}

\section{Introduction}

Driving simulator is a simulated equipment which can simulate the action of vehicle driving correctly and can obtain the same feeling of real vehicle driving in the main characteristics. It is a simulated system with the purpose of man - machine interaction, based on computers. The simulated driving simulator needs to not only build a realistic simulated environment but also simulated the vehicle mobile behavior.

In order to simulate all kinds of the vehicle mobile behaviors exactly, we need to analyze different terrain affecting the vehicle mobile behavior, get the change of the maximum speed, average vehicle speed, maximum acceleration, turning radius in different environments and establish the precise vehicle model. At the same time, we should establish the exact physical and geometric algorithm according to Newton mechanics theorem instead of only according to the scripts movement, so that the vehicle can move in line with true physical laws. However, writing the complex algorithm of Physics will consume the Developers' much energy and reduce the development efficiency.

Therefore, This paper uses the Open Dynamics Engine and the Open Scene Graph to build a realistic virtual driving environment, analyzing kinds of terrain impacts on vehicle mobile behavior, making the vehicles move in simulated environment in line with the law of real world.

\section{The Introduction of ODE}

Because the vehicle mobile behavior relates to a collision between the rolling, sliding, and object, and some reactions, it's difficult to simulate these complex behavior through predetermined script. We can simulate the vehicle mobile behavior vividly by that physic engine gives vehicles corresponding physical properties and does mechanical simulation on behaviors between Vehicle movement and collision with the virtual scene.

This paper uses physic engine ODE to simulate vehicle behaviors. ODE is a high-performance simulation reservoir of open rigid body dynamics., using the collision detection system of hard collision mode inside and preventing the traditional phenomenon. It also offers advanced joint types and is fit for simulation of the vehicle motion. 


\section{Analysis of factors influencing vehicle}

An important evaluation criteria of driving simulator is the similarity between it and the real equipment. It needs to build a realistic simulated environment, what's more, it also simulates the diverse behaviors of vehicle in real world so that the virtual vehicle in it can move as the equipment does in real word.

As for the vehicle mobile behavior in training simulator, it shows different movement performance under the effect of all kinds of terrain environment factors. So we need to analyze different factors affecting vehicle movements and build exact the equations of motion.

The vehicle is mainly affected by gravity, ground support force, the vehicle traction force, air resistance, gradient resistance when it drives in straight line and we analyze the force situation when the vehicle drives in straight line, the one in driving direction will be mainly researched .The stress equation could be simplified as equation 2.1:

$$
\mathrm{F}=\mathrm{F}_{\mathrm{t}}-\mathrm{F}_{\mathrm{w}}-\mathrm{F} \omega^{-} \mathrm{Ff}
$$

In this equation, $F_{t}$ stands for the vehicle traction force, $F w$ for the air resistance, $F_{\omega}$ for the gradient resistance and $F_{f}$ for the ground drag. Only when the vehicle traction force is bigger than the air resistance, gradient resistance and the ground drag, could the vehicle drive normally on the ground. The magnitude of these forces are not only determined by the vehicle condition and performance but also are influenced by the terrain conditions.

equation 2.2 is according to the Newton's laws :

$$
\mathrm{F}=\mathrm{M} d t
$$

We could find that in the driving direction of the vehicle, the influence of vehicle's force condition on its mobile behavior is mainly reflected in the magnitude of acceleration. Besides, the vehicle's performance and traffic capacity suffer different influences under different terrain conditions.

The influence of the slope of ground resistance

Different slope sizes result in different slope resistance and this affects stress in the direction of vehicle's moving.

In the equation (2.1) : the size of $\mathrm{F}_{\omega}$ (slope resistance) can be expressed by the equation 2.3

$$
\mathrm{F}_{\omega}=\mathrm{Mg}^{*} \sin \alpha
$$

In equation (2.3), $\mathrm{M}$ is for vehicle quality, $\mathrm{g}$ is for the acceleration of gravity, $\alpha$ is for slope angle.

slope angle affects not only $\mathrm{F}_{\omega}$ (slope resistance) , thus affecting acceleration during vehicle's moving ,but also vehicle capacity. On different slopes, the speed is different, and different

\begin{tabular}{|c|c|c|c|c|c|c|}
\hline & \multirow{2}{*}{$\begin{array}{l}\text { Types of } \\
\text { vehicles }\end{array}$} & \multicolumn{4}{|c|}{$\begin{array}{l}\begin{array}{l}\text { Speeds under different slope } \\
(\mathrm{Km} / \mathrm{h})\end{array} \\
\end{array}$} & \multirow{2}{*}{$\begin{array}{l}\text { Extreme } \\
\text { slope } \\
\text { angle }\end{array}$} \\
\hline & & $3 \sim 6^{\circ}$ & $6 \sim 10^{\circ}$ & $10 \sim 15^{\circ}$ & $15 \sim 20^{\circ}$ & \\
\hline & $\begin{array}{l}\text { wheeled } \\
\text { vehicle }\end{array}$ & $20 \sim 15$ & $15 \sim 12$ & $12 \sim 8$ & $5 \sim 3$ & $-30^{\circ}$ \\
\hline & $\begin{array}{l}\text { track } \\
\text { vehicle }\end{array}$ & $12 \sim 10$ & $10 \sim 7$ & $7 \sim 5$ & $5 \sim 3$ & $17 \sim 25^{\circ}$ \\
\hline \multirow{5}{*}{ Table2.2 spee } & under & \multicolumn{2}{|c|}{ Altitude $(\mathrm{m})$} & \multicolumn{2}{|c|}{ speed $(\mathrm{Km} / \mathrm{h})$} & \multirow{5}{*}{ different attitude } \\
\hline & & \multicolumn{2}{|c|}{0} & \multicolumn{2}{|r|}{100} & \\
\hline & & \multicolumn{2}{|c|}{1000} & \multicolumn{2}{|r|}{88.6} & \\
\hline & & \multicolumn{2}{|c|}{2000} & \multicolumn{2}{|r|}{78.1} & \\
\hline & & \multicolumn{2}{|c|}{3000} & \multicolumn{2}{|r|}{68.5} & \\
\hline
\end{tabular}
properties of the vehicle can go through different maximum slope. The table.2.1 is about different speeds of different vehicles on different slopes . 
On the other hand, in equation (2.1) the size of $\mathrm{F}_{\mathrm{f}}$ (ground resistance), can be expressed by the equation 2.4:

$$
F_{F}=\mu M g \& \cos a
$$

From equation (2.4), we can know slope angle affects Ground support force。The smaller the slope angle is ,the larger the ground support force will be. The ground resistance will be larger when the ground resistance coefficient is certain.

The effect of elevation on the vehicle traction power

Altitude effects on vehicle traction is mainly on the influence of vehicle engine power output. the $\mathrm{F}_{\mathrm{t}}$ in equation (2.1) expressed by the equation 2.5:

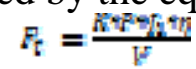

$\mathrm{K}$ is often parameters in equation (2.5), $\mathrm{P}$ for engine power (KW), $\mathrm{f} 1 \mathrm{for}$ correction coefficient of atmospheric pressure on the impact of change on engine power for machinery total power, $\mathrm{V}$ is velocity $(\mathrm{Km} / \mathrm{h})$.

With the increase of altitude, the air pressure reduced. The decreases of air density result in reduction of air during vehicle engine work. The fuel cannot complete be burned, in(2.5) the f1 attenuate continuously, reducing engine power and the vehicle traction power. The biggest speed of Vehicles will decrease as the altitude increases.

the table.2.2 is the vehicle speed changes under different altitude (altitude is 0 when the speed is 100$)$.

The ground soil influence on the ground resistance.

From equation(2.4) shows that the ground resistance of $\mathrm{Ff}$ in addition to the affection by the slope, also affected by the ground resistance coefficient.

Ground resistance coefficient is mainly influenced by surface soil . If the surface soil is different, ground resistance coefficient will be different, this will affect the distance of acceleration and the braking distance when the vehicle is running on different surface soil.

when a vehicle turns, the ground that provides the centripetal force is affected by the ground resistance coefficient. When a vehicle turns, and the speed and the slope is set, the greater the ground resistance coefficient, the smaller the minimum turning radius. The table.2.3 shows the influence of soil on the ground resistance coefficient.

Table .2.3 influence of soil on the ground resistance coefficient

\begin{tabular}{|l|l|l|l|}
\hline Ground soil & $\begin{array}{l}\text { coefficient of } \\
\text { resistance }\end{array}$ & Ground soil & $\begin{array}{l}\text { coefficient of } \\
\text { resistance }\end{array}$ \\
\hline cement & $0.03-0.04$ & snowfiled & $0.10-0.25$ \\
\hline asphalt & $0.03-0.05$ & dry sand & $0.15-0.18$ \\
\hline $\begin{array}{l}\text { unsurfaced } \\
\text { road }\end{array}$ & 0.1 & $\begin{array}{l}\text { Frozen } \\
\text { groud }\end{array}$ & $0.03-0.04$ \\
\hline gravel & 0.065 & paddy field & $0.15-0.25$ \\
\hline wet sand & 0.1 & wetland & $0.15-0.25$ \\
\hline
\end{tabular}

Based on introduction of the ODE simulation process, we are able to set up a physical world that obeys the law of Newtonian mechanics, the object can move according to the Newton's laws of motion. But due to the physics engine is based on the physical description of these objects (not graphics) and the interaction of simulation of the movement, it doesn't care about how to display its object. And the movement and state of objects cannot be displayed. Due to the ODE and OSG is based on frame cycle, this makes the possibility of combining both. 
To combines physics engine and graphics rendering engine, the graphics rendering engine creates graphical model with physics engine to create physical model, the physical properties of a need to create two identical physical world and graphics world, the physical model and graphic model one to one correspondence, by updating the rotation matrix and real-time synchronization update, the attitude of the virtual object and direction with calculation result of physics engine drive graphics rendering engine mapping and rendering. The specific process as shown in figure 3.1:

Fig 3.1 process of combining Physics engine and graphics rendering engine

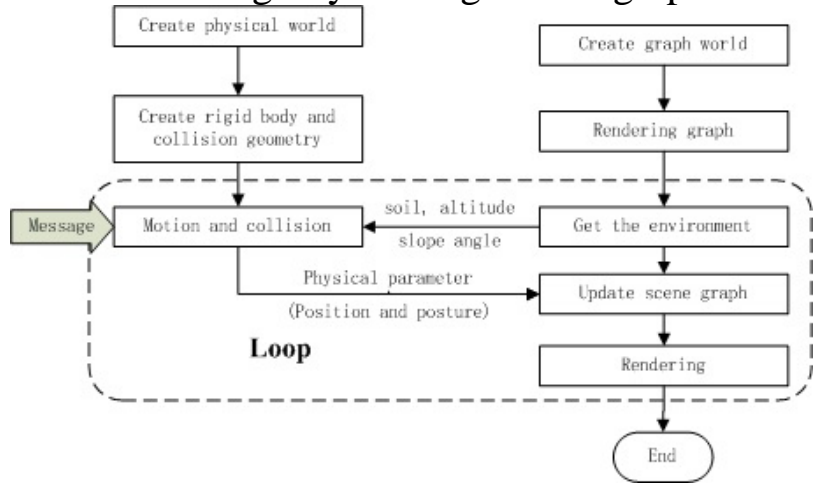

1To create the physical world, and accept the graphics rendering engine mapping the geometry of the initial position and posture of creationof the corresponding rigid body and collision geometry and initialization

2, physics engine receives the message from outside, drive the rigid motion and the collision detection in the physical world, and record the position and posture. itself.

3 , the graphics in the graphics world model receiving record physical model parameters update

4, graphics engine for rendering and drawing, and it can output eventually through graphics hardware equipment on the display

\section{Design and implementation of the program}

Lifelike physical model is the core of the behavior of vehicles, vehicle displayed by graphics rendering engine, physics engine to solve the physical model of the vehicle position and posture is the key to the behavioral simulation.

Because the ODE is used for simulating the rigid body motion, so the vehicle's physical model mainly divided into the body and wheels, its geometric properties based on OBB bounding box, car body and wheels with hinge2 joint connection. So you set up a physical model of the vehicle. Corresponding a CVehicle class should be established, the physical properties of vehicles, terrain factors and encapsulate all kinds of driving operation. Here mainly introduce CVehicle class member variables:

class CVehicle: public osg::Group

\{

//physical property

dMass m_dCarMass;

dMass m_dWheelMass;

dReal m_rMaxSpeed;

dReal m_rTorque;

dReal m_dBreakTorque;

//terrain influence

char* Soil; 
double m_dDamp;

double m_dHeight;

\}

Program structure is to use a loop, using the physical model of continuously updated graphics model motion, achieve the movement synchronization, constantly updated per frame, to produce continuous motion picture .

In each frame, the vehicle will detect its place belongs to that category of terrain , access to external influence factors such as altitude, soil, and on the basis of the maximum velocity of ground resistance factor, vehicle such as variable is given the corresponding values, and into the relevant equation, adjust the vehicle's motion.

\section{Conclusion}

Combining with physics engine the ODE and OSG rendering graphics engine, through the analysis of terrain environment on the impact of various factors on the vehicle, has realized the vehicle in different terrain simulation environment under the influence of motor behavior.

Through professional physics engine for simulating vehicle behavior, and lifelikely reflects not only the vehicle of climbing, turning, etc, also greatly reduced the workload of collision detection and collision process. Based on physical simulation method is applied to virtual environment, to make the authenticity of virtual environment and greatly improve interaction and it allows developers to focus more on external factors such as terrain environment effects and improvement of the vehicle model.

\section{References}

[1]Ou Yanghuiqing, Chen Fumin..Research and implementation of binding of physics engine and graphic rendering engine[J], Computer Engineering and Design,2008,29(11):5580-5582,5620

[2]Lin Hui, Gong Jian-hua. On Virtual Geographic Environments[J], ACTA GEODAETICA et CARTOGRAPHICA SINICA[J], 2002,31(1):1- 6.

[3]Lin Hui, Gong Jian-hua,Shi Jing-jing. From Maps to GIS and VGE-A Discussion on the Evolution of the Geographic Language[J].Geograhy and Geo-Information Science[J]. ,2003, 19 (4) :18- 23.

[4]You Ren,Chen Ding-fang. Research on Physical Simulation and Application of Physical Engine in Virtual Environment[J],Journal of Hubei University of Technology,2008,23(2):7-9.

[5] Philips J,Schneider David $\mathrm{H}$ Eberly.geometric tools for computer graphic [M].Beijing.Publishing House of Electronics Industry, 2005.

[6] Jef Craighead,Robin Murphy,Jenny Burke,et a1.A survey of

commercial\& open source unmaned vehicle simulators[C].Roma, Italy: IEEE Intemational Conference on Robotics and Automation.2007: 852, 854-855.

[7] ODE Main Page . OPEN DYNAMIC ENGINE[EB / OL] • http : / / http://ode-wiki.org/wiki/index.php?title=Main_Page.

[8]Wang Xuan.Research on Motion Simulation and Collision Detect for distributed Virtual Driving Simulator based on OSG[D].Wuhan: Wuhan University of Technology,2008.9.

[9]Chen Ding-fang, Yin Nian-dong,Li Xun-xiang.The Distributed Interactive Vehicles Simulation System for Driving Training[M].Beijing:Science Press,2009:29.

[10]Wang Li-gong. An New Interactive Augmented Reality System[J],Techniques of Automation and application,2010,29(7):36-38. 